\title{
Optimum acceptability of telecommunications networks: a multi-criteria approach
}

\author{
María Romero María Luisa Cuadrado Luis Romero \\ Carlos Romero
}

\begin{abstract}
This paper proposes an aggregated index of acceptability that can establish a "ranking" of the possible candidate hub cities in the design of a telecommunications network. The main advantages of the aggregated index proposed are the following: (a) it takes into account several wide-ranging design criteria, (b) its formulation is given by a lineal mathematical expression, (c) the procedure for calculating the index is very simple, and (d) the candidate hubs can be ranked differently depending on the aggregation structure of the criteria involved. Taking this considerations, the application of the proposed methodology can supplement the information supplied by more sophisticated multicriteria approaches based generally upon $0 / 1$ goal programming. The theory presented is illustrated using a case study taken from the literature.
\end{abstract}

Keywords Multiple criteria decision making · Telecommunications networks · Hubs selection 


\section{Introduction}

The problem of designing a telecommunications network over a given geographically dispersed area is an important practical problem that involves engineering and economic aspects (see Kawasumi et al. 2007; Lee et al. 2001; Nazem et al. 1994). Most efforts undertaken along these lines resort to binary mathematical programming. In fact, the value of a generic decision variable $x_{k}$ is 1 if the $\mathrm{k}$-th hub point is chosen; otherwise the value of $x_{k}$ is 0 . Consequently, an objective function reflecting the interests of the decision maker is optimised subject to the satisfaction of a set of constraints that the network design should meet. Although undoubtedly worthwhile, this approach to the problem is not exempt of difficulties. Thus, the choice of the objective function to be optimised is generally problematic. Indeed, the reliability of designing a telecommunications network is actually a multidimensional problem, involving several, wide-ranging criteria. In this context, it is very difficult to arrive at a good design solution just by optimising a single criterion. For instance, a candidate hub that minimises the implementation budget may apply to a very low populated area, which may not be acceptable. This problem has been successfully addressed by reformulating the mathematical programming model commented above as a binary goal programming. Lee et al. (1996) pioneered work in this direction (see Lee et al. 1994) where a similar methodology is applied to an analogous problem consisting of the design of a files system).

The other problem underlying the binary mathematical programming approach is that the system that is going to be designed is usually huge. Therefore, the size of the binary mathematical programming problem in real-world situations can lead to a sizeable computational burden (see Lee et al. 1994; Nazem et al. 1994, 1996). Moreover, the solution of the model provides the "optimum design", but it does not state how acceptable each potential hub is, which can be problematic in some cases.

This paper proposes a straightforward methodology (see Roy and Slowinsk 2013; Steuer 1989) that is able to attach an index of acceptability to each candidate hub by means of a simple computation. This index will take into account all the points of view (i.e., criteria) that the decision maker considers that affects the decision on the acceptability of a potential hub point. Therefore, it will sort (strong or weak) the $n$ potential hub points so that the overall utility of the telecommunications network is maximized. The proposed method does not claim to be an alternative to the approaches based upon binary mathematical programming. It is an attempt to provide, with a small computational burden, additional information that complements the output of these approaches.

\section{Analytical procedure}

\subsection{Definition of model inputs}

Let us consider $\mathrm{i}=1,2, \ldots, \mathrm{n}$ potential candidate hubs in the design of a telecommunication network. Each candidate hub is evaluated according to $\mathrm{j}=1,2$, 
.., $\mathrm{m}$ criteria/acceptability indicators. In the case of a rural telecommunications network, as recommended by the Federation of American Scientists, (see Lee et al. 1996) the possible acceptability indicators to be applied to each candidate hub are: economic activity, population, construction and operation costs, education, transportation, etc.

The problem posed in this paper will involve defining an index of acceptability that aggregates the different acceptability indicators attached to each one of the $n$ potential hub locations. Therefore, the $\mathrm{n}$ potential hub locations can be ranked according to their overall acceptability. If the formulation is to be of pragmatic interest, the aggregate index should satisfy some common sense properties, such as:

(a) Since the number of candidate hubs and acceptability indicators in many realworld situations is very large, the index calculation procedure should be very simple.

(b) The engineering and economic interpretation of the index should be clear and straightforward.

(c) The index should represent a good balance between very wide-ranging indicators.

The first step is to introduce the following notation.

$\mathrm{P}_{\mathrm{ij}}=$ performance achieved by the $\mathrm{i}$-th candidate hub $(\mathrm{i}=1,2, \ldots, \mathrm{n})$ when it is evaluated according to the $j$ th indicator of acceptability $(j=1,2, \ldots, m)$.

$P_{j}^{*}=$ ideal value of the $j$-th indicator of acceptability (i.e. the best value possible).

This value is obtained by optimizing each indicator of acceptability individually and is given by maximum performance if the indicator is of the type "more is better" (e.g., economic activity) or by minimum performance if the indicator is of the type "less is better" (e.g., implementation budget).

$\mathrm{P}_{* \mathrm{j}}=$ anti-ideal value achieved by the $\mathrm{j}$-th indicator of acceptability (i.e. the worst value possible). This value is obtained by optimizing, in the opposite direction as in the previous case, each indicator of acceptability individually and is given by minimum performance if the indicator is of the type "more is better" or by maximum performance if the indicator is of the type "less is better".

$\overline{\mathrm{P}}_{\mathrm{ij}}=$ normalised performance achieved by the $\mathrm{i}$-th candidate hub when it is evaluated according to the $\mathrm{j}$-th indicator of acceptability.

$\mathrm{w}_{\mathrm{j}}=$ weight measuring the relative importance attached to the $\mathrm{j}$-th indicator of acceptability. By hypothesis it is assumed that $w_{j}>0, j=1,2, \ldots, m$.

$I_{i}^{A}=$ aggregate value index of acceptability for the generic $i$-th candidate hub.

Given that the indicators of acceptability are mostly measured in different units (e.g., operating costs in monetary units, health care according to a qualitative index, etc.), the first and absolutely necessary step for constructing the aggregated index of acceptability is to normalise the different performances achieved by the candidate hubs considered. A simple and pragmatic normalisation procedure successfully used in other contexts (see Díaz-Balteiro and Romero 2004) for an ecological context) is as follows: 


$$
\bar{P}_{i j}=\frac{P_{i j}-P_{* j}}{P_{j}^{*}-P_{* j}}, \quad i=1,2, \ldots, n, \quad j=1,2, \ldots, m
$$

With the normalisation given by (1), the normalised performance values $\overline{\mathrm{P}}_{\mathrm{ij}}$ are dimensionless, positive and bounded between 0 (when the candidate hub achieves the anti-ideal value) and 1 (when the candidate hub achieves the ideal value), respectively. Having this in mind and indicated by $\mathbf{P}^{*} \in \mathbf{R}^{\mathrm{m}}$ the ideal vector (vector whose components are the ideal values of $\mathrm{m}$ acceptability criteria) and by $\mathbf{P}_{*} \in \mathbf{R}^{\mathrm{m}}$ the anti-ideal vector (vector whose components are the anti-ideal values of $\mathrm{m}$ acceptability criteria), with the normalization made, we can verify that:

$$
\mathbf{P}^{*}=\mathbf{1}=(1,1, \ldots, 1)^{\mathrm{T}} \quad \text { and } \quad \mathbf{P}_{*}=\mathbf{0}=(0,0, \ldots, 0)^{\mathrm{T}}
$$

that is, the ideal vector is equal to $\mathbf{1}$ and the anti-ideal vector is equal to $\mathbf{0}$, which is very convenient for undertaking any possible aggregation and allows to easily evaluate the achievement percentage of each criterion with respect to its ideal value.

\subsection{Vector of the criteria weights}

Among the different possible methodologies, based on pairwise comparison matrices, of computing the vector of the criteria weights (see Choo and Wedley 2004; Gonzalez- Pachon and Romero 2007, 2014; Havanov et al. 2008; Lin 2007), we propose to obtain this applying the Dominant Eigen vector Method (EVM) to the square matrix $A=\left(a_{i j}\right)$ of order $m$, positive $\left(a_{i j}>0\right)$, of diagonal elements one $\left(a_{i i}=1\right)$, reciprocal $\left(a_{i j} \cdot a_{j i}=1\right)$ and generally inconsistent $\left(a_{i j} \neq a_{i k} \cdot a_{k j}\right)$, which is obtained by pair-wise comparison of the $m$ criteria being considered. This method, developed by Saaty to calculate local priorities in the Analytic Hierarchy Process (AHP) (see Saaty 1980, 1995, 2011, 2012; Vaidya and Kumar 2006), evaluates the weight vector by resolving the following system of equations:

$$
A \mathbf{w}=\lambda_{\max } \mathbf{w}, \quad \sum_{\mathbf{j}=1}^{\mathrm{m}} \mathrm{w}_{\mathrm{j}}=1
$$

where $\lambda_{\max }$ is the maximum eigenvalue of matrix $\mathrm{A}$.

In this paper, the preferred method for weighing the criteria is using the EVM, as it allows to easily evaluate the consistency of the value judgments made by the decision maker when constructing the pairwise comparison matrix, analysing the proximity of the values $m$ (order of the matrix) and $\lambda_{\max }$ (maximum eigenvalue of the matrix), verifying that if these values match, the matrix will be consistent.

In the practical application of this method, due to its operational difficulty, for the algebraic calculation of eigenvalues and eigenvectors, the use of Expert-Choice software is recommended; this not only helps to obtain an estimation of the weight vector with a tolerable level of inconsistency, but also to rapidly and easily do a sensitivity analysis that guarantees the stability of the weight obtained against small changes in the value judgments issued on building the pairwise comparison matrix of the acceptability indicators considered. The development of Expert-Choice has 
been supervised by Saaty and its application covers a wide range of practical experiences in diverse fields.

\subsection{Aggregate index of acceptability}

Known the preferential weights $w_{\mathbf{j}}>0, \mathrm{j}=1,2, \ldots, \mathbf{n}$ and the values $\overline{\mathrm{P}}_{\mathrm{ij}}, \mathrm{i}=1,2$, $\ldots, \mathbf{n}, j=1,2, \ldots, m$ to establish the aggregated index of acceptability $\mathrm{I}_{i}^{\mathrm{A}}$ of each of the $\mathrm{n}$ candidate hubs considered and given that for the inherent conflict of multiple criteria the ideal solution is infeasible (it is only a point of reference for the decision marker), we are going to accept (see Andre and Romero 2008) the following decision rule or behavioural axiom:

"Candidate hubs that are closer to the ideal are preferred to candidate hubs that are further away from the ideal"

This axiom introduced in the decision theory by Zeleny $(1974,1982)$ is a postulate of behavior firmly rooted in the psychology that allows to hierarchically sort the alternatives of choice, taking into account the preferences of the decision maker. To make this axiom operational in our contest it is necessary to define in $\mathbf{R}^{\mathbf{m}}$ a general distance function between the vector of the normalised performance achieved by the generic i-th candidate hub $\overline{\mathbf{P}}_{\mathrm{ij}}=\left(\overline{\mathrm{P}}_{\mathrm{i} 1}, \overline{\mathrm{P}}_{\mathrm{i} 2}, \ldots, \overline{\mathrm{P}}_{\mathrm{im}}\right)^{\mathrm{T}}$ and the ideal vector $\mathbf{1}=(1,1, \ldots, 1)^{\mathrm{T}}$ With this purpose in mind (see Erhgott and TenfeldePodehl 2003), the following distance functions are introduced:

$$
\mathrm{L}_{\mathrm{p}}\left(\mathbf{1}, \overline{\mathbf{P}}_{\mathrm{ij}}\right)=\left[\sum_{\mathrm{j}=1}^{\mathrm{m}} \mathrm{w}_{\mathrm{j}}^{\mathrm{P}}\left(1-\overline{\mathrm{P}}_{\mathrm{ij}}\right)^{\mathrm{p}}\right]^{\frac{1}{p}}, \quad 1 \leq \mathrm{p} \leq \infty .
$$

In the definition, based in Minkowski's metric, of the distance function given; we have considered that, with the normalization of acceptability indicators of the candidate hubs performed, it is possible to simplify their formulation and write it, regardless of the absolute value of the addends contained in their expression.

It is important to emphasize here that:

1. The distance function (2) is not used in its geometric sense, but as a proxy measure for human preferences; in this sense, it is possible to give values to metric $\mathrm{p}$ in the closed interval $[1, \infty]$.

2. The metric $\mathrm{p}$ is a control parameter that, taking into account the preferences of the decision marker, allows to obtain aggregated or balanced solutions with respect to the realization of the indicators of acceptability considered, verifying that it is advisable to select a small value of $p$ when we obtain a solution that minimizes the sum of the deviations and a high value of $p$ when the interest lies in obtaining a solution that gives more importance to the individual deviations.

Specifying the metric $\mathrm{p}$ in (2), the value of the aggregated index of acceptability for the $\mathrm{i}$-th candidate hub, is given by the expression: 


$$
\mathrm{I}_{\mathrm{i}}^{\mathrm{A}}=\mathrm{L}_{\mathrm{p}}\left(\mathbf{1}, \overline{\mathbf{P}}_{\mathrm{ij}}\right)
$$

By particularising the distance function (2) for the metrics $\mathrm{p}=1$ and $\mathrm{p}=\infty$ the following results are obtained:

$$
\begin{aligned}
& \text { Distance Manhattan }:=\mathrm{L}_{\mathbf{1}}\left(\mathbf{1}, \overline{\mathbf{P}}_{\mathrm{ij}}\right)=\sum_{\mathrm{j}=1}^{\mathrm{m}} \mathrm{w}_{\mathbf{j}}\left(1-\overline{\mathrm{P}}_{\mathrm{ij}}\right)
\end{aligned}
$$

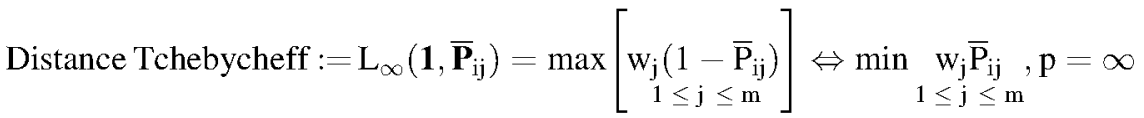

With respect to these distance functions, defined by lineal mathematical expressions, it is interesting to note that:

- The first expression adds the normalized and weighted degrees of proximity of all the criteria to their ideal value; verifying that, when the metric $p$ increases, more importance is given to the bigger deviations; whereas, the second is limited to finding the maximum degree of normalized and weighted proximity of all criteria to their ideal value.

- The solution that assigns the lowest numerical value to the distance for $\mathrm{p}=1$ $(:=$ compromise solution for $\mathrm{p}=1)$ is a solution of maximum utility, i.e. the solution that maximises the normalized and weighted sum of the obtained values for the acceptability indicators considered.

- The solution that assigns the lowest numerical value to the distance for $p=\infty$ $(:=$ compromise solution for $p=\infty)$ is a solution of maximum equality, i.e. the solution that maximises the balance between the achievements of the different acceptability indicators considered.

- The compromise solutions for $p=1$ and $p=\infty$ define a subset called compromise set, and the solutions for other values of metric $\mathrm{p}$ generally belong to this set (see Blasco et al. 1999; Yu 1973 for a justification of this crucial property of the compromises sets).

Taking this into account, in order to calculate in a computationally simple way the aggregate acceptability index $\mathrm{I}_{i}^{\mathrm{A}}$ given, generally (i.e. for all values to the metric $\mathrm{p}$ belonging to the open interval $(1, \infty)$ ) by nonlinear expression (3); we propose to calculate it (see Andre and Romero 2008; Romero 2004; Steuer 1989), evaluating the following lineal convex combination:

$$
\left.\left.(1-\lambda) \mathrm{L}_{\mathrm{p}}\left(\mathbf{1}, \overline{\mathbf{P}}_{\mathrm{ij}}\right)\right]_{\mathrm{p}=\infty}+\lambda \mathrm{L}_{\mathrm{p}}\left(\mathbf{1}, \overline{\mathbf{P}}_{\mathrm{ij}}\right)\right]_{\mathrm{p}=1}, \quad \lambda \in[0,1]
$$

Specifying in this expression the value of $\lambda$ and considering (4) the aggregated acceptability index for the $\mathrm{i}$-th candidate hub, results in the following: 


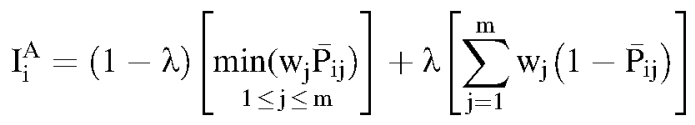

It is important to note that:

- The aggregate indexes of acceptability given by the formula (6) for values $\lambda=1$ and $\lambda=0$ are the same as those given by the formula (2) for the metrics $p=1$ and $\mathrm{p}=\infty$, respectively.

- In the expression (6), analogously to $\mathrm{p}$ in (3), $\lambda$ is a control parameter that is not only used to obtain different rankings of the candidate hubs, but more importantly it reflects different structures of preference of the decision maker with respect to the selection of candidate hubs.

\subsection{Hierarchical sorting of candidates}

The application of the Zeleny axiom to the set of the aggregated index of acceptability $\left\{\mathrm{I}_{\mathrm{i}}^{\mathrm{A}}, \mathrm{i}=1,2, \ldots, \mathbf{n}\right\}$ obtained applying (6) to each $\mathbf{n}$ candidate hubs considered, analogously to the TOPSIS method (see Behzadlan et al. 2012), we will be able to hierarchically sort them for selection purposes (see Lei et al. 2012; Romero and Rehman 2003). In this way, taking into account that with the normalisation system used the biggest value of $\overline{\mathrm{P}}_{\mathrm{ij}}, \forall \mathrm{i}, \mathrm{j}$ is equal to 1 , the first candidate hub of the hierarchy (i.e., the most acceptable candidate hub in the metric $\mathrm{p}$ specified) will be the one with the lowest value in the calculated aggregated index of acceptability (i.e. the candidate hub that minimises the distance between the vectors $\overline{\mathbf{P}}_{\mathrm{ij}}$ and $\mathbf{1}$ ).

Below, we will analyse the different structures of preference to select the best candidate hub corresponding to the values $\lambda=1$ and $\lambda=0$.

The "most acceptable candidate hub" for $\lambda=1$ is the candidate hub with the best aggregated achievement, that is, the candidate hub that maximises the weighted sum of the normalised acceptability indicators. This additive solution is the "best" in aggregate terms, but may be unacceptable in practical terms. In fact, a big aggregated average may be compatible with a very poor performance of one of the indicators (e.g. economic activity), which can make the candidate in question ineligible in economic and/or engineering terms.

The "most acceptable candidate hub" for $\lambda=0$ is the candidate hub for which the deviation of the indicator most displaced with respect to the ideal value (i.e., 1) is minimized. Therefore, the candidate hub maximising the balance between the achievements of the different acceptability indicators considered, is the "best".

In many situations, neither of the two previous candidate hubs will be acceptable because the "most acceptable" candidate hub in one case $(\lambda=1)$ may be one for which the performance of one of the indicators is very poor, and the "most acceptable" candidate hub for the other case $(\lambda=0)$ will be very balanced but may have a poor aggregate performance. 
One procedure for overcoming the above difficulties is to interpret the control parameter $\lambda$ as a device for making a trade-off between "maximum aggregate acceptability" (efficiency) and "most balanced acceptability" (equity). Thus, compromises or intermediate solutions, with sensible properties in terms of good aggregate performance and good balanced performance can be obtained assigning values to the parameter $\lambda$ belonging to the open interval $(0,1)$; which will be specified performing a sensitivity analysis with the Expert-Choice program, choosing one of the five possibilities of sensitivity analysis: Evaluation sensitivity, Dynamic sensitivity, Gradient sensitivity, Two dimensions graph and Sensitivity of weighted differences, that this program offers.

Hereunder a simple but illustrative example referring to the design of a telecommunications network (i.e., the selection of candidate hubs) will be used to assess the operational, as well as the pragmatic value of the proposed methodology.

\section{An illustrative example}

A case study, reported by Lee et al. (1996), will be used to demonstrate the operation as well as the suitability of the proposed index. The purpose of this example is to design a telecommunications network in the state of Nebraska, where 24 candidate hubs (cities) are considered. Six acceptability indicators are involved in the design problem. The indicators are as follows: Economic activity, population, budget, health care, education and transportation. Lee et al. (1996) arrived at an optimum telecommunications design by formulating and solving a $0 / 1$ goal programming model. We are going to apply the methodology proposed in this paper to rank the 24 candidate hubs according to the value achieved by the aggregated index of acceptability given for the expression (6).

Table 1 (see Lee et al. 1996, p. 41) shows the values of acceptability indicators achieved by each of the 24 candidate hubs when they are evaluated according to each of the six acceptability indicators considered.

Table 2 shows the normalised values of the acceptability indicators. These values are obtained by applying the normalisation procedure defined by (1) to the values of Table 1. Remember that in this context zero denotes the anti-ideal value, while one denotes the ideal value.

On the other hand, the relative importance attached to each indicator of acceptability by the decision maker was elicited by Lee et al. (1996) applying Dominant Eigenvector Method (EVM) to the square matrix $A$ : $\left(a_{i j}\right)$ of order six, obtained by pairwise comparison of the six acceptability indicators considered (see Lee et al. 1996, p. 41), given for: 
Table 1 Values of acceptability indicators achieved by each candidate hub. (see Lee et al. 1996, p. 41)

\begin{tabular}{|c|c|c|c|c|c|c|}
\hline Candidate hub & $\begin{array}{l}\text { Number of } \\
\text { employees } \\
\text { Increase (unit: } \\
10,000 \text { ) }\end{array}$ & $\begin{array}{l}\text { Counties } \\
\text { population } \\
\text { Increase } \\
\text { (unit: } 10,000 \text { ) }\end{array}$ & $\begin{array}{l}\text { Budget } \\
\text { Decrease } \\
\text { (unit: } \\
\$ 10,000 \text { ) }\end{array}$ & $\begin{array}{l}\text { Health } \\
\text { care } \\
\text { Increase } \\
\text { index }\end{array}$ & $\begin{array}{l}\text { Education } \\
\text { Increase } \\
\text { index }\end{array}$ & $\begin{array}{l}\text { Transportation } \\
\text { Increase index }\end{array}$ \\
\hline 1. Alliance & 0.27 & 1.42 & 25 & 1 & 4 & 3 \\
\hline 2. Blair & 0.32 & 1.55 & 20 & 1 & 4 & 3 \\
\hline 3. Beatrice & 0.55 & 3.58 & 20 & 5 & 2.5 & 3 \\
\hline 4. Chadron & 0.18 & 0.94 & 40 & 1 & 6 & 3 \\
\hline 5. Columbus & 1.01 & 2.96 & 15 & 1 & 4 & 4 \\
\hline 6. Falls City & 0.19 & 1.10 & 10 & 1 & 2 & 3 \\
\hline 7. Fremont & 1.04 & 3.58 & 8 & 4 & 4 & 4 \\
\hline $\begin{array}{l}\text { 8. Gering/ } \\
\text { Scottsbluff }\end{array}$ & 1.07 & 3.84 & 20 & 5 & 4 & 3 \\
\hline $\begin{array}{l}\text { 9. Grand } \\
\text { Island }\end{array}$ & 1.94 & 4.99 & 8 & 5 & 4 & 4 \\
\hline 10. Hastings & 1.06 & 3.11 & 10 & 5 & 5 & 4 \\
\hline 11. Holdredge & 0.30 & 1.01 & 10 & 1 & 2 & 4 \\
\hline 12. Kearney & 1.17 & 3.79 & 5 & 5 & 7 & 6 \\
\hline 13. Lexington & 0.53 & 2.23 & 20 & 5 & 2 & 6 \\
\hline 14. Lincoln & 8.18 & 20.30 & 7.5 & 10 & 10 & 9 \\
\hline 15. McCook & 0.34 & 1.30 & 45 & 1 & 4 & 3 \\
\hline $\begin{array}{l}\text { 16. Nebraska } \\
\text { City }\end{array}$ & 0.36 & 1.50 & 18 & 1 & 2 & 4 \\
\hline 17. Norfolk & 1.24 & 3.23 & 20 & 5 & 4 & 3.5 \\
\hline $\begin{array}{l}\text { 18. North } \\
\text { Platte }\end{array}$ & 0.74 & 3.47 & 10 & 4 & 4 & 6 \\
\hline 19. Ogallala & 0.25 & 0.92 & 10 & 1 & 2 & 6 \\
\hline 20. Omaha & 21.32 & 41.03 & 2 & 10 & 9 & 10 \\
\hline 21. Sydney & 0.22 & 1.01 & 50 & 1 & 4 & 6 \\
\hline $\begin{array}{l}\text { 22. South } \\
\text { Sioux City }\end{array}$ & 0.69 & 1.72 & 7.5 & 1 & 4 & 5 \\
\hline 23. Wayne & 0.19 & 0.98 & 22 & 1 & 6 & 3 \\
\hline 24. York & 0.46 & 1.50 & 10 & 2 & 4 & 5 \\
\hline
\end{tabular}


Table 2 Normalised values of indicators of acceptability achieved by each candidate hub

\begin{tabular}{lllllll}
\hline Candidate hub & $\begin{array}{l}\text { Number of } \\
\text { employees }\end{array}$ & $\begin{array}{l}\text { Counties } \\
\text { population }\end{array}$ & Budget & $\begin{array}{l}\text { Health care } \\
\text { index }\end{array}$ & $\begin{array}{l}\text { Education } \\
\text { index }\end{array}$ & $\begin{array}{l}\text { Transportation } \\
\text { index }\end{array}$ \\
\hline 1. Alliance & 0.0042 & 0.0120 & 0.5208 & 0 & 0.2500 & 0 \\
2. Blair & 0.0066 & 0.0157 & 0.6250 & 0 & 0.2500 & 0 \\
3. Beatrice & 0.0175 & 0.0663 & 0.6250 & 0.4444 & 0.0625 & 0 \\
4. Chadron & 0 & 0.0005 & 0.2083 & 0 & 0.5000 & 0 \\
5. Columbus & 0.0393 & 0.0508 & 0.7290 & 0 & 0.2500 & 0.1428 \\
6. Falls City & 0.0005 & 0.0045 & 0.8333 & 0 & 0 & 0 \\
7. Fremont & 0.0407 & 0.0663 & 0.8750 & 0.3333 & 0.2500 & 0.1428 \\
8. Gering/ & 0.0421 & 0.0728 & 0.6250 & 0.4444 & 0.2500 & 0 \\
Scottsbluff & & & & & & \\
9. Grand & 0.0833 & 0.1015 & 0.8750 & 0.4444 & 0.2500 & 0.1428 \\
Island & & & & & & \\
10. Hastings & 0.0416 & 0.0546 & 0.8333 & 0.4444 & 0.3750 & 0.1428 \\
11. Holdredge & 0.0057 & 0.0022 & 0.8333 & 0 & 0 & 0.1428 \\
12. Kearney & 0.0468 & 0.0715 & 0.9375 & 0.4444 & 0.6250 & 0.4286 \\
13. Lexington & 0.0166 & 0.0327 & 0.6250 & 0.4444 & 0 & 0.4286 \\
14. Lincoln & 0.3784 & 0.4832 & 0.8854 & 1 & 1 & 0.8571 \\
15. McCook & 0.0076 & 0.0095 & 0.1042 & 0 & 0.2500 & 0 \\
16. Nebraska & 0.0085 & 0.0144 & 0.6667 & 0 & 0 & 0.1428 \\
City & & & & & & \\
17. Norfolk & 0.0501 & 0.0576 & 0.6250 & 0.4444 & 0.2500 & 0.0714 \\
18. North & 0.0265 & 0.0636 & 0.8333 & 0.3333 & 0.2500 & 0.4285 \\
Platte & & & & & & \\
19. Ogallala & 0.0033 & 0 & 0.8333 & 0 & 0 & 0.4285 \\
20. Omaha & 1 & 1 & 1 & 1 & 0.8750 & 1 \\
21. Sydney & 0.0019 & 0.0022 & 0 & 0 & 0.2500 & 0.4285 \\
22. South & 0.0241 & 0.0199 & 0.8854 & 0 & 0.2500 & 0.2857 \\
Sioux City & & & & & & \\
23. Wayne & 0.0005 & 0.0015 & 0.5833 & 0 & 0.5000 & 0 \\
24. York & 0.0132 & 0.0144 & 0.8333 & 0.1111 & 0.2500 & 0.2857 \\
\hline
\end{tabular}

$$
A=\left(\begin{array}{llllll}
1 & 2 & 3 & 5 & 6 & 7 \\
& 1 & 1 & 3 & 5 & 5 \\
& & 1 & 3 & 4 & 6 \\
& & & 1 & 3 & 5 \\
& & & & 1 & 2 \\
& & & & & 1
\end{array}\right)
$$

Accordingly, the weights obtained were as follows: $\mathrm{w}_{1}=0.396, \mathrm{w}_{2}=0.215, \mathrm{w}_{3}=0.202, \mathrm{w}_{4}=0.103, \mathrm{w}_{5}=0.050, \mathrm{w}_{6}=0.034$. 


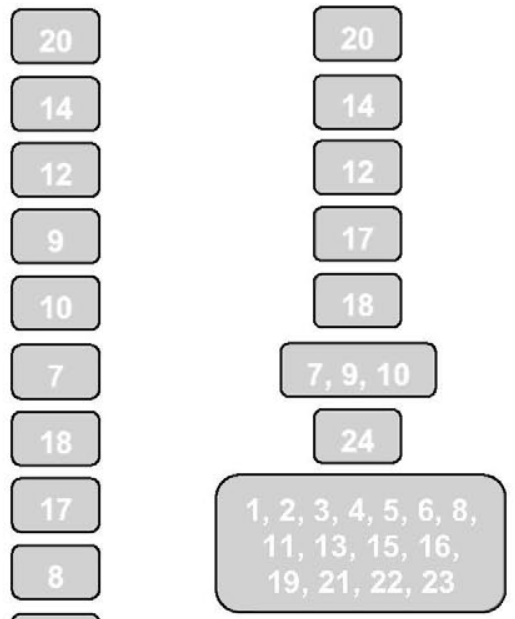

22 (b)
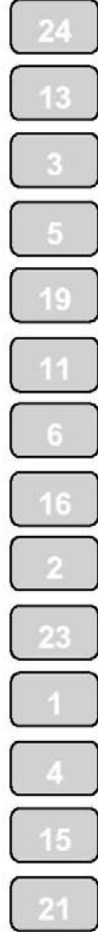

(a)

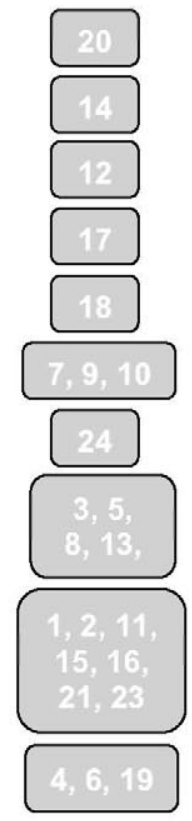

(c)

Fig. 1 Rankings of hub cities candidates. a Maximum aggregate achievement $(\lambda=1)$. b. Maximum balanced achievement $(\lambda=0)$. c Maximum balanced achievement $(\lambda=0)$, taking into account number of zeros 
Different rankings of selection can be obtained by applying the aggregated index of acceptability given by expression (6) to the above data. Figure 1 shows the two extreme "rankings" obtained; that is, the ranking with the "best aggregate achievement" (i.e., $\lambda=1$ ) and the ranking with the "most balanced achievement" (i.e., $\lambda=0$ ).

It should be noted that the candidate hubs for which at least one indicator of acceptability achieves the anti-ideal value appear at the bottom of the "most balanced ranking". It is obvious that for these hubs, the value of the respective aggregate index of acceptability is zero. A sensible step, however, would be to discriminate the level of acceptability according to the number of zeros for each candidate hub (i.e., the number of acceptability indicators achieving the anti-ideal value). Hence, the indifference set $\{1,2,3,4,5,6,8,11,13,15,16,19,21,22,23\}$ is partitioned into the following three subsets: $\{3,5,8,13,22\}$ with one zero, $\{1,2,11,15,16,21,23\}$ with two zeros and, finally, $\{4,6,19\}$ with three zeros. That is, for $\lambda=0$ ("best balance"), the poorest candidate hubs are the geographical locations 4, 6 and 19, since three acceptability indicators achieve the anti-ideal values for these hubs. This last "weak ordering" appears in the Fig. 1(c). It is interesting to note that the only two candidate hubs (cities) not selected in the exercise described in Lee et al. (1996) are precisely 4 (Chadron) and 6 (Falls City), that is, two of the worst candidate hubs obtained in our exercise from the balanced point of view.

\section{Concluding remarks}

The design of a telecommunications network over a given geographically dispersed area, taking into account several criteria/indicators, is now an important decisionmaking problem. So far, this type of problem has been addressed using different methodologies not exempt of difficulties. These include the computational burden, as well as the additive character of the process of aggregating the different criteria involved. The aggregate index of acceptability that is proposed in this paper, based on the use of the $\lambda$ parameter, can circumvent some of these difficulties and can be used to hierarchically sort any number of possible candidate hubs. In fact, the computation of the index is very simple and the "additive" case is just one of the different options for aggregating the considered criteria. Consequently, the generation of a "ranking" of candidate hubs, according to an aggregate index of acceptability, is a very useful piece of information in many real-world situations.

\section{References}

Andre FJ, Romero C (2008) Computing compromise solutions: on the connections between compromise programming. Appl Math Comput 195:1-10

Behzadlan M, Otaghsara SK, Yazdani M, Ignatius J (2012) A state of art survey of TOPSIS applications. Expert Syst Appl 39(17):13051-13069 
Blasco F, Cuchillo-Ibáñez E, Alonso-Morón M, Romero C (1999) On the monotonicity of the compromise set in multicriteria problems. J Optim Theory Appl 102:69-82

Bu L, Van Duin JHR, Wiegmans B, Luo Z, Yin C (2012) A selection of city distribution locations in urbanized areas. Procedia Soc Behav Sci 39:556-567

Choo EU, Wedley WC (2004) A common framework for deriving preference values from pairwise comparison matrices. Comput Oper Res 31:893-908

Díaz-Balteiro L, Romero C (2004) In search of a natural systems sustainability index. Ecol Econ 49:399-403

Erhgott M, Tenfelde-Podehl D (2003) Computation of Ideal and Nadir values and implications for their use MCDM methods. Eur J Oper Res 151:119-139

Gonzalez-Pachon I, Romero C (2007) Inferring consensus weights from pairwise comparison matrices without suitable properties. Ann Oper Res 154:123-132

González-Pachón J, Romero C (2014) Analysis of pairwise comparison matrices. In: Wiley enciclopedia of operations research and management science, pp 1-9

Havanov N, Kolari J, Sokolov M (2008) Deriving weights from general pairwise comparison matrices. Math Soc Sci 55:205-220

Kawasumi Y, Miyoski Y, Koizumi K (2007) How to provide broadband access for rural and remote areas of developing countries. In: Proceedings of PTC'07, Telecom with vision. Honolulu, Hawaii

Lee H, Shi Y, Stolen J (1994) Allocating data files over a wide area network: goal setting a compromise design. Inf Manag 26:85-93

Lee H, Shi Y, Nazem S (1996) Supporting rural telecommunications: a compromise solution approach. Ann Oper Res 68:33 45

Lee H, Shi Y, Nazem S, Kang SY, Park TH, Sohn MH (2001) Multicriteria hub decision making for rural telecommunications networks. Eur J Oper Res 133:483-495

Lin C (2007) A revised framework for deriving preferences values from pairwise matrices. Eur J Oper Res 176:1145-1150

Nacem S, Shi Y, Lee H, Sung Yeol K, Tae Ho P, Myung S (2001) Multicriteria hub decision making for rural area telecommunication networks. Eur J Oper Res 133(3):483-495

Nazem S, Liu Y, Shi Y (1994) Designing rural areas telecommunication network via hub cities. Omega 22:305-314

Nazem S, Liu Y, Lee H, Shi Y (1996) Implementing telecommunications infrastructure: a rural America case. Telematics Inform 13:23-31

Romero C, Rehman T (2003) Multiple criteria analysis for agricultural decision, vol 11. Elsevier Science, New York

Romero C (2004) A general structure of achievement function for a goal programming model. European Journal of Operation Resesearch 153:675-686

Roy B, Slowinsk R (2013) Questions guiding the choice of multicriteria decision aiding method. EURO J Decis Process 1:69-97

Saaty TL (1980) Multicriteria decision making: the analytic hierarchy process. McGraw-Hill, New York

Saaty TL (1995) Decision making for leaders: the analytic hierarchy process for decision in a complex world. RWS Publications, Pittsburgh

Saaty TL (2011) Decision making with the analytic network process: economic, political, social and technological applications with benefits opportunities costs and risks. Springer, New York

Saaty TL (2012) Models, methods, concepts and applications of the decision making for analytic hierarchy process. Springer, New York

Steuer RE (1989) Multiple criteria optimization: theory, computation and application. Drieger, Malabar Vaidya OS, Kumar S (2006) Analytic hierarchy process: an overview of applications. Eur J Oper Res $169: 1-29$

Yu PL (1973) A Class of solutions for group decision problems. Manag Sci 19:936-946

Zeleny M (1974) A concept of compromise solutions and the method of the displaced ideal. Comput Oper Res 1:479-496

Zeleny M (1998) Multiple criteria decision making: Eight concepts of optimality. Hum Syst Manag 17(2):97-107 